\title{
Theory of mind, emotion recognition, delusions and the quality of the therapeutic relationship in patients with psychosis - a secondary analysis of a randomized-controlled therapy trial
}

Stephanie Mehl ${ }^{1,2^{*}}$ (D, Klaus Hesse ${ }^{3}$, Anna-Christine Schmidt ${ }^{1}$, Martin W. Landsberg ${ }^{1}$, Daniel Soll², Andreas Bechdolf ${ }^{4,5}$, Jutta Herrlich ${ }^{6}$, Tilo Kircher², Stefan Klingberg ${ }^{3}$, Bernhard W. Müller ${ }^{7}$, Georg Wiedemann $^{8}$, Andreas Wittorf ${ }^{3}$, Wolfgang Wölwer ${ }^{9}$ and Michael Wagner ${ }^{1}$

\begin{abstract}
Background: Cognitive models of psychosis postulate an important role of Theory of mind (ToM) in the formation and maintenance of delusions, but research on this plausible conjecture has gathered conflicting findings. In addition, it is still an open question whether problems in emotion recognition (ER) are associated with delusions. We examined the association of problems in ToM and ER with different aspects of delusions in a large sample of patients with psychosis enrolled in a therapy trial. This also enabled us to explore the possible impact of ToM and ER on one part of patients' social life: the quality of their therapeutic relationship.

Methods: Patients with psychotic disorders and delusions and/or hallucinations $(n=185)$ and healthy controls $(n=$ 48) completed a ToM picture sequencing task and an ER task. Subsequently, patients were enrolled in a randomized-controlled Cognitive Behavior Therapy (CBT) trial (ISRCTN29242879). Patients and therapists rated the quality of the therapeutic relationship during the first five sessions of therapy.

Results: In comparison to controls, patients were impaired in both ToM and ER. Patients with deficits in ER experienced more severe delusional distress, whereas ToM problems were not related to delusions. In addition, deficits in ER predicted a less favorable therapeutic relationship and interactional problems viewed by the therapist. Impaired ER also moderated (increased) the negative influence of delusions on the therapeutic relationship and interactional difficulties viewed by the therapist.

Conclusions: Cognitive models on the formation and maintenance of delusions should consider ER as a potential candidate that might be related to the formation and maintenance of delusional distress, whereas problems in ToM might not be directly related to delusions and secondary dimensions of delusions. In addition, problems in ER in patients with psychosis might have an impact on the quality of the therapeutic relationship and patients with problems in ER are more likely to be viewed as problematic by their therapists. Nevertheless, training ER might be a way to improve the quality of the therapeutic relationship and potentially the effectiveness of CBT or other interventions for patients with psychosis.
\end{abstract}

Keywords: Schizophrenia, Delusions, Theory of mind, Emotion recognition, Quality of the therapeutic relationship, Interactional problems

\footnotetext{
* Correspondence: stephanie.mehl@staff.uni-marburg.de

'Department of Psychiatry and Psychotherapy, University of Bonn,

Sigmund-Freud-Straße 25, 53105 Bonn, Germany

${ }^{2}$ Department of Psychiatry and Psychotherapy, Philipps-University of

Marburg, Rudolf-Bultmann-Straße 8, 35039 Marburg, Germany

Full list of author information is available at the end of the article
}

(c) The Author(s). 2020 Open Access This article is distributed under the terms of the Creative Commons Attribution 4.0 International License (http://creativecommons.org/licenses/by/4.0/), which permits unrestricted use, distribution, and reproduction in any medium, provided you give appropriate credit to the original author(s) and the source, provide a link to the Creative Commons license, and indicate if changes were made. The Creative Commons Public Domain Dedication waiver (http://creativecommons.org/publicdomain/zero/1.0/) applies to the data made available in this article, unless otherwise stated. 


\section{Background}

Theory of Mind (ToM) is defined as the ability to attribute other peoples' mental states, intentions and emotions and to understand and predict their behaviour [1]. It is part of the concept of social cognition, i.e. the perception, processing and interpretation of social signals $[2,3]$. Frith [4] proposed that deficits in ToM are a predisposing factor for persecutory delusions. Based on this assumption, ToM in patients with psychosis was found to be more than one standard deviation below the performance of healthy controls $[5,6]$. In addition, several studies found evidence of an association between ToM deficits and persecutory [7] and general delusions [8]. Consequently, ToM has been incorporated into theoretical models as one causal factor involved in the formation and maintenance of delusions $[9,10]$ and positive symptoms of psychosis [11].

Nevertheless, in a recent review, ToM problems were associated with more severe general delusions or delusions of persecution in about half of all studies that addressed this question, whereas the other studies did not find an association [12]. Consequently, several newer theoretical models excluded ToM problems as a causal factor for persecutory delusions or positive symptoms $[12,13]$.

One explanation for the inconsistent results could be the small sample size of most studies investigating associations between ToM and delusions that was also not mended by the latest meta-analysis: rather than assessing the relation between ToM and delusions the authors investigated the relationship of ToM and reality distortion (an aggregation of delusions and hallucinations) and did not find any evidence for an association [14].

In addition, it is possible that problems in ToM are rather a risk factor for psychosis in general than delusions, whereas more basal problems of patients with psychosis in emotion recognition (ER), the ability to identify other persons' emotions by using emotionally salient information in the environment (verbal and non-verbal cues) [15] might be more important in the formation and maintenance of delusions. This is suggested by the fact that patients with psychosis are severely impaired in ER in comparison to controls $[16,17]$ and by the results of the meta-analysis mentioned above that suggest an association between problems in ER and reality distortion [14, 18].

Further, as other social-cognitive biases as the jumping to conclusions-bias are related with more severe delusional frequency and delusional distress [19], it is possible that problems in ToM and ER problems might also enhance the frequency and distress caused by delusions, this question has not been addressed until today.

While an associations with delusions is unclear, ToM problems in patients with psychosis are closely related to problems with their social performance such as inadequate eye contact, speech modulation and conversational flow [20], interpersonal difficulties [21, 22] and problems in social functioning [23, 24]. Nevertheless, it is unclear whether problems in ToM and ER in patients with psychosis also affect the quality of their social interactions in real life.

One paradigm of a social interaction in real life is the therapeutic alliance defined as affective bond and consensus with regard to goals and treatment tasks [25]. As the therapeutic alliance is highly important for the effectiveness of Cognitive Behavior Therapy (CBT [26-28]), it is crucial to assess whether problems in ToM and ER might have a negative effect on it. This question has been investigated in one study reporting associations between ToM problems and patients' ratings on the quality of the therapeutic alliance [29]. With regard to problems in ER, their impact on the therapeutic alliance has not been addressed until today, but an impact might be possible. If either ToM problems and/or deficits in ER might be associated with the therapeutic relationship, Cognitive Behavior Therapy (CBT) should more strongly address patients' problems in ToM and ER to improve the therapeutic alliance and consequently its effectiveness.

Besides TOM and ER abilities, persecutory delusions also negatively impact the therapeutic alliance in CBT [30]. Interestingly, preserved ToM abilities were found to moderate (reduce) the negative influence of persecutory delusions on social functioning [31]. Thus, it is likely that positive ToM and ER performance might protect patients from the additional negative impact of delusions on the quality of the therapeutic relationship. Patients with preserved ToM and ER performance might be more able to understand the intentions and emotions of their therapists correctly and act accordingly, while still assuming that other persons are to be mistrusted. But, the question whether intact ToM and ER performance might reduce (moderate) the negative impact of delusions on the therapeutic relationship has not been assessed until today.

The present study was a secondary analysis of a randomised-controlled therapy trial [32] and was set out to investigate whether problems in ToM and ER are more severe in patients with psychosis (in comparison to controls (hypothesis 1)) and whether they are related to delusions and secondary dimensions of delusions (frequency and distress) in a large patient sample (hypothesis 2). Both hypothesis 1 and 2 have been pre-specified in the trial proposal. In an additional exploratory analysis, the study aimed to investigate, whether problems in ToM and ER influence the quality of the therapeutic relationship (hypothesis 3). Further, we assessed in an additional exploratory analysis, whether positive ToM and ER performance moderate the association between delusions and the quality of the therapeutic relationship (hypothesis 4). 


\section{Methods}

\section{Subjects}

Subjects were 185 patients with psychosis and 48 healthy controls from the "Cognitive behavioural therapy for persistent positive symptoms (CBTp) in psychotic disorders" Trial [32] (ISRCTN29242879), a multi-centred randomized controlled trial investigating the efficacy of CBT for patients with psychosis in comparison to supportive therapy.

Inclusion criteria were a diagnosis of a psychotic disorder (schizophrenia $(n=147)$, schizophreniform disorder $(n=1)$, schizoaffective disorder $(n=25)$ or delusional disorder $(n=12))$ assessed with the Structured Clinical Interview for DSM-IV (SCID [33]).

Further inclusion criteria were persistent positive symptoms for at least the last three months and a minimum score of four in the item P1 (delusions in general: $n=162$ ) and/or in the item P3 (hallucinations: $n=79$; both: $n=56$ ) of the Positive and Negative Syndrome Scale (PANSS [34]), age between 18 and 59, adequate language fluency and a verbal intelligence quotient $>80$ in the German IQ test Mehrfachwahl-Wortschatztest (MWT-B [35]. Exclusion criteria for healthy controls were mental disorders in their lifetime as assessed with the Structured Clinical Interview for DSM-IV (SCID [33]).

Patients were recruited from six different psychiatric centers (Bonn, Cologne, Duesseldorf, Duisburg, Frankfurt am Main, Tuebingen, Germany); healthy controls were recruited in all six centers via public advertisement and matched with regard to age, gender, and education to the first 48 patients that were already recruited.

From the initial study sample $(n=330)$, a small number of patients dropped out before they were asked to participate $(n=9)$ or refused to participate in the additional assessment $(n=22)$, had problems with their eyesight $(n=3)$, with German language $(n=4)$, with test instructions $(n=14)$ or decided to participate in a nested fMRI study instead $(n=93)$. There were no statistically significant differences between the patients $(n=185)$ who performed in ToM and ER in the present study and patients who participated in the fMRI paradigm and between patients who refused to be tested and patients who endorsed testing with regard to sociodemographic and clinical variables (all $p>.10$ ).

All participants were informed about the assessment and gave written informed consent. In case of a legal guardian, the patients and the guardian were informed about the assessment and both the patient and the guardian gave written informed consent. The ethics committees at the six centres' medical faculties approved the study. The randomized-controlled trial and the secondary analysis presented here adhere to Consort criteria.
From the total patient sample, a smaller number of patients participated in at least three therapy sessions and these patients were included in analyses regarding the association between ToM, ER, delusions and the therapeutic relationship ( $n=174$, CBT: $n=90$, ST: $n=84)$.

\section{Measures}

Theory of Mind (ToM) performance was assessed using a cartoon task paradigm $[36,37]$ that presented excellent test-retest reliability with regard to activation of key ToM areas in previous studies [38] Participants were asked to view 14 comic strips presented on a computer screen in pseudo-randomized order. Each comic strip included two phases: in Phase I, three pictures (3 $\mathrm{s}$ each) depicting an unfolding story plot were shown sequentially. In Phase II, two answer pictures were presented simultaneously (26 s), and participants were asked to choose the picture showing the logical ending of the story. The comic strips presented a social interaction between two protagonists (e.g. a person asks a second person for a glass of water). In order to solve the task, participants were required to infer the intentions of the characters correctly. The sum scores of correct answers were used as measure for ToM (range between 0 and 14).

Emotion Recognition (ER) was assessed with a Pictures of Facial Affect test (PFA). 28 faces (10 photographs selected from Ekman and Friesen's pictures of facial affect [39] and 18 photographs from a comparable set of pictures $[40,41])$ served as stimuli depicting four basic emotions (fear, anger, disgust and sadness). Each emotion was displayed by seven different faces showing each emotion once. Faces were presented sequentially in randomized order and participants were asked to select the most applicable emotion from a multiple-choice list. The PFA sum score of correct answers was used as measure of ER (ER total score; range between 0 and 28). In addition, scores of the four negative emotions were also used (ER fear, ER anger, ER sadness and ER disgust; ranges between 0 and 7).

The Positive and Negative Syndrome Scale (PANSS [34]) is a semi-structured interview assessing 30 symptoms divided into three scales (positive scale, negative scale, general psychopathology scale, ranges between 7 and 14 for the positive and negative scale and between 16 and 30 for the general psychopathology scale) using a 7 -point Likert scale. PANSS ratings were performed by trained raters who received ten training sessions in all items. The inter-rater reliability (ICC, corr. $\mathrm{R}^{2}$ ) was satisfactory to high (between .86 and .92). The item P1 (general delusions) was used as assessment of general delusions and the item P6 as assessments of delusions of persecution (range of both items between 1 and 7).

The Psychotic Symptom Rating Scale (PSYRATS [42]) is a semi-structured interview with six items assessing 
different dimensions of delusional beliefs such as amount of preoccupation, duration of preoccupation, conviction, disruption of daily life, amount of distress and intensity of distress on a 5-point-Likert scale (scores between 0 and 4). Inter-rater-reliability and validity were high in a sample of patients with psychosis [42]. Based on results of a factor analysis [43], the items are summed up to the subscale PSYRATS delusional frequency (sum of the subscales amount of preoccupation, duration of preoccupation, conviction and disruption of daily life, range between 0 and 16) and PSYRATS delusional distress (sum of the subscales amount of distress and intensity of distress, range between 0 and 8 ).

The quality of the therapeutic relationship was assessed using the short versions of the Patient Session Questionnaire (PSQ) and the Therapist Session Questionnaire (TSQ) derived from the German Berner Therapist- and Patient- Session Questionnaire [Berner Patienten- und Therapeutenstundenbogen 2000]. Internal consistency (Cronbach's alpha) was found to be good and effect sizes of correlations between quality of the therapeutic relationship viewed by the patient and therapist were generally large [44]. Both therapists and patients answered the questionnaires after the first five sessions of either CBT or ST. In order to reduce socially more desirable answers, patients answered the questionnaire after every session and put their ratings in a closed box.

From the PSQ, the quality of the therapeutic alliance viewed by the patient subscale (PSQ therapy alliance) was used that consists of the sum of three items that were answered by patients after a therapeutic session on a 7-point-Likert scale (scores between -3 and +3 ) and reflect the quality of the therapeutic relationship viewed by the patient ("The therapist and I understand each other", "Today I felt at ease with the therapist", "I think the therapist is really interested in my well-being"). In addition, from the TSQ, the 3-item subscale TSQ therapist alliance was used that measures the mean score of the quality of the therapeutic relationship viewed by the therapist. Items are answered on a 6-point-Likert scale ("The patient and I understand each other." "Today I felt at ease with the patient." "The patient and I work on joint goals."; scores between -3 and +3 ). The mean score over the first five sessions was used (range between -3 and +3 ).

In addition, we used one item in the TSQ covering interpersonal difficulties viewed by the therapist (TSQ interactional difficulties: "I believe this patient is difficult in terms of his/her interaction") that was answered on a 7-point Likert scale (scores between - 3 and +3$)$. Again, mean scores of the first five sessions were used (range between -3 and +3 ).

\section{Statistical analysis}

First, we used Fisher's exact tests, $C h i^{2}$ tests and $t$-tests in order to compare patients with schizophrenia and controls in socio-demographic, clinical variables and ToM / ER problems (hypothesis 1). Exploration of the raw data showed ceiling effects for ToM and ER (total score and scores of the specific four negative emotions) as defined by Uttl [45] Thus, due to the skewedness of the data, we used an arcus-sinus-transformation as recommended by [46] that allows application of parametric statistics.

Hypothesis 2 (associations between ToM, ER and delusions) was assessed using Pearson's bivariate correlations between ToM, ER (ER total score, ER fear, ER anger, ER sadness and ER disgust) and delusions in general (PANSS item P1), delusions of persecution (PANSS item P6) and the two PSYRATS subscales delusional frequency and delusional distress. Spearman rank correlations were used to examine hypothesis 3 (associations between ToM, ER total score, ER fear, ER anger, ER sadness and ER disgust and the therapeutic relationship), as PSQ and TSQ subscales were not normally distributed.

A series of hierarchical regression analyses were performed to assess whether ToM and ER problems moderate the association between delusions and the quality of the therapeutic relationship (hypothesis 4). All measures that were bivariately associated with the quality of the therapeutic relationship (PSQ therapy alliance / TSQ therapist alliance / TSQ interactional difficulties), were meancentered and then included as predictors in the first block of the hierarchical regression analysis and their interaction term in the second block. General symptom severity (PANSS total score) was included as covariate.

Significant moderating relationships were analysed using the Johnson-Neyman technique included in the PROCESS macro [47], a follow-up method for regressions containing interaction coefficients that enables to identify over what range of the moderator a predictor has significant versus non-significant effects on the outcome measure [48].

\section{Results}

Table 1 shows socio-demographic and clinical data of patients with psychosis and healthy controls. There were no statistically significant differences between both groups in terms of age or gender. Compared to controls, patients showed significantly lower verbal intelligence scores (MWTB), but associations between ToM, ER and verbal intelligence were small and not statistically significant and no statistical adjustment for verbal intelligence was performed.

\section{Group differences in ToM and ER (hypothesis 1: pre- specified analysis)}

With regard to hypothesis 1 , results revealed that patients with psychosis presented more pronounced 
Table 1 Sociodemographic and clinical variables of patients with psychosis and healthy controls

\begin{tabular}{|c|c|c|c|c|}
\hline & $\begin{array}{l}n \text { (patients/ } \\
\text { Controls) }\end{array}$ & $\begin{array}{l}\text { Patients with } \\
\text { psychosis } \\
(n=185)\end{array}$ & $\begin{array}{l}\text { Healthy controls } \\
(n=48)\end{array}$ & Test statistics \\
\hline & & $n(\%) / M(S D)$ & $n(\%) / M(S D)$ & \\
\hline Age (ys.) & $185 / 48$ & $38.62(9.78)$ & $35.69(9.44)$ & $t(231)=1.865, p=.063$ \\
\hline Gender (fem.) & $185 / 48$ & $107(57.84 \%)$ & $20(41.68 \%)$ & ${ }^{\mathrm{a}} p=1.000$ \\
\hline School education: & $185 / 48$ & & & $X^{2}(4)=8.331, p=.080$ \\
\hline 13 years & & $97(52.43 \%)$ & $24(50 \%)$ & \\
\hline 10 years & & $56(30.27 \%)$ & $22(45.83 \%)$ & \\
\hline 9 years & & $31(16.76 \%)$ & $2(4.17 \%)$ & \\
\hline Less than 9 years & & $1(0.54 \%)$ & - & \\
\hline Verbal intelligence (MWT-B) ${ }^{b}$ & $176 / 46^{b}$ & $107.48(14.82)$ & $114.52((15.36)$ & $t(222)=-2.85, p=.005$ \\
\hline Duration of illness & 185 & $15.36(9.63)$ & & \\
\hline Theory of Mind (ToM) ${ }^{c}$ & $185 / 39^{c}$ & $12.56(1.74)$ & $13.31(1.00)$ & $t(222)=-2.711, p=.007$ \\
\hline ER total score & $185 / 45^{d}$ & $23.31(3.23)$ & $24.8(2.06)$ & $t(228)=-2.954, p=.003$ \\
\hline ER fear & $185 / 45^{d}$ & $5.90(1.43)$ & $6.22(0.90)$ & $t(228)=-1.456, p=.147$ \\
\hline ER anger & $185 / 45^{d}$ & $6.05(1.19)$ & $6.69(0.56)$ & $t(228)=-3.512, p=.001$ \\
\hline ER sadness & $185 / 45^{d}$ & $6.28(1.15)$ & $6.33(1.09)$ & $t(228)=-.275, p=.783$ \\
\hline ER disgust & $185 / 45^{d}$ & $5.09(1.28)$ & $5.56(1.16)$ & $t(228)=-2.247, p=.026$ \\
\hline PANSS positive scale & 185 & $17.71(3.79)$ & & \\
\hline PANSS negative scale & 185 & $13.57(4.17)$ & & \\
\hline PANSS general psychopathology scale & 185 & $32.62(7.27)$ & & \\
\hline PANSS P1 delusions in general & 185 & $4.44(1.02)$ & & \\
\hline PANSS P6 delusions of persecution & 185 & $3.37(1.57)$ & & \\
\hline PSYRATS delusional frequency & 185 & $8.76(3.56)$ & & \\
\hline PSYRATS delusional distress & 185 & $5.1(2.46)$ & & \\
\hline TSQ therapy alliance (therapist) & $174^{d}$ & $1.09(.65$ & & \\
\hline TSQ interactional difficulties (therapist) & $174^{d}$ & $-.50(1.23)$ & & \\
\hline PSQ therapy alliance (patient)sessions 1-5 & $174^{d}$ & $1.72(.62)$ & & \\
\hline
\end{tabular}

Notes: ${ }^{a}$ Fishers Exact Test; ${ }^{\mathrm{b}} n=9$ patients did not perform in the MWT-B due to language problems and two controls due to organisational problems, ${ }^{\mathrm{c}} n=9$ controls did not participate at the ToM assessment, ${ }^{d} n=3$ controls did not participate at the ER assessment; ER: Emotion recognition; PANSS: Positive and Negative Syndrom Scale; PSYRATS: Psychotic Symptoms Rating Scale; TSQ therapy alliance = Therapist session questionnaire = quality of therapeutic relationship viewed by the therapist (mean score sessions1-5); TSQ interactional difficulties = Therapist Session Questionnaire rating of interactional difficulties rated by the therapist (mean score sessions 1-5), PSQ therapy alliance = Patient Session Questionnaire rating of the quality of the therapeutic relationship rated by the patient (mean score sessions 1-5); ${ }^{\mathrm{d}}$ TSQs were filled by 13 different therapists and 174 patients

deficits in both ToM and ER (descriptive scores are depicted in Fig. 1). With regard to ER, patients were more impaired than controls in the total ER score and in recognizing the emotions anger and disgust, whereas there were no statistically significant differences between both groups with regard to recognizing the emotions fear and sadness.

\section{Associations between ToM, ER and delusions (hypothesis 2: pre-specified analysis)}

As depicted in Table 2, there was no statistically significant association between ToM problems and any measure of delusions. However, problems in ER were related with more pronounced PSYRATS delusional distress. Specifically, delusional distress (PSYRATS delusional distress) showed a statistically significant association with the ability to recognize the emotions fear and disgust (ER fear and ER disgust), whereas the two other specific ER scores (ER anger and ER distrust) were not associated with delusional distress. Further, neither the ER total score nor the four specific negative emotions scores were related to any other measure of delusions.

Associations between ToM, ER, delusions and the quality of the therapeutic relationship (hypothesis 3: exploratory analysis)

Patients' ratings of the quality of the therapeutic relationship (PSQ therapy alliance) were not related with ToM nor ER total score and ER subscales (see Table 3). In addition, there was no statistically significant association 


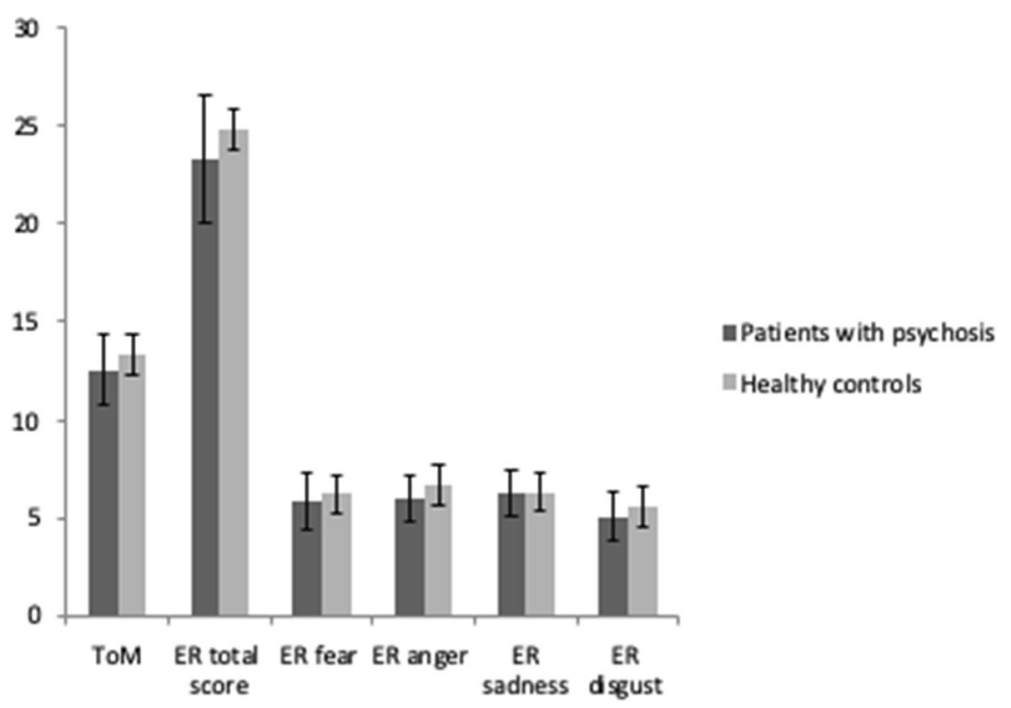

Fig. 1 ToM and Emotion Recognition in patients with psychosis and healthy controls. Notes: ToM = Theory of Mind, ER=Emotion Recognition; Patients with psychosis: $n=185$; Healthy controls: Theory of Mind: $n=42$; Emotion Recognition: $n=39$, error bars display the standard deviation

between PSQ therapy alliance and all other measures of delusions. Therapists' ratings of the quality of the therapeutic relationship (TSQ therapy alliance) were related to ER total score and to all four ER subscales (ER fear, ER anger, ER sadness and ER disgust) and PSYRATS delusional frequency. TSQ interactional problems showed a statistically significant association with ER total score and PSYRATS delusional frequency. In addition, TSQ interactional problems were related with problems in recognizing the emotions fear and disgust (ER fear, ER disgust), whereas there were no statistically significant associations between TSQ interactional problems and problems in recognizing anger and sadness (ER anger and ER sadness).

Moderation analyses (hypothesis 4: exploratory analysis) As can be seen in Table 4, including ER and PSYRATS delusional frequency in the first block of the hierarchical regression analysis predicting TSQ therapist alliance resulted in a statistically significant model, but solely ER was a statistically significant predictor of TSQ therapist alliance. Including the interaction term in the second block resulted in a statistically significant model and the interaction explained additional variance in TSQ therapy alliance, suggesting a statistically significant moderation effect.

The Johnson-Neyman technique revealed that ER was predictive of TSQ therapy alliance for participants with ER scores lower than $20.45 \%$ and above 79.65\% (all $p<0.05$ ). This suggests that in patients with scores in ER in percentiles between zero and 20.45, ER problems attenuated the negative influence of delusional frequency on TSQ therapy alliance and in patients with ER scores in percentiles above $79.65 \%$, good ER ability protected them from the

Table 2 Results of Pearson correlation analyses between Theory of Mind, emotion recognition and delusions in the patient sample $(n=185)$

\begin{tabular}{|c|c|c|c|c|c|c|}
\hline & ToM & ER total score & ER fear & ER anger & ER sadness & ER disgust \\
\hline ER total score & $.316^{* *}$ & & & & & \\
\hline ER fear & $.207^{* *}$ & $.743^{* *}$ & & & & \\
\hline ER anger & $.244^{* *}$ & $.462^{* *}$ & .052 & & & \\
\hline ER sadness & $.171^{*}$ & $.688^{* *}$ & $.479^{* *}$ & .230 & & \\
\hline ER disgust & $.228^{* *}$ & $.690^{* *}$ & $.389^{* *}$ & .057 & $.356^{* *}$ & \\
\hline PANSS P1 delusions in general & .096 & .006 & .014 & .096 & -.100 & -.033 \\
\hline PANSS P6 delusions of persecution & .113 & .021 & -.012 & .101 & -.019 & .048 \\
\hline PSYRATS delusional frequency & -.057 & .068 & .064 & .070 & -.028 & .018 \\
\hline PSYRATS delusional distress & .020 & $.219^{* *}$ & $.166^{*}$ & .085 & .124 & $.236^{* *}$ \\
\hline
\end{tabular}

Notes: ER Emotion recognition, PANSS Positive and Negative Syndrom Scale, PSYRATS Psychotic Symptoms Rating Scale, *: $p<.05 ; *^{* *}: p<.01$ 
Table 3 Results of Spearman correlation analyses between Theory of Mind, emotion recognition and the quality of the therapeutic relationship in the patient sample

\begin{tabular}{llll}
\hline & $\begin{array}{l}\text { PSQ therapy alliance viewed } \\
\text { by the patient }(n=174)\end{array}$ & $\begin{array}{l}\text { TSQ therapy alliance viewed } \\
\text { by the therapist }(n=174)\end{array}$ & $\begin{array}{l}\text { TSQ interactional difficulties } \\
\text { viewed by the therapist }(n=174)\end{array}$ \\
\hline PSQ therapy alliance & - & $.240^{* *}$ & $-.222^{* *}$ \\
TSQ therapist alliance & $.240^{* *}$ & - & $-.701^{* *}$ \\
TSQ interactional difficulties & $-.222^{* *}$ & $-.701^{* *}$ & - \\
ToM & -.014 & .112 & -.077 \\
ER total score & -.039 & $.347^{* *}$ & $-.278^{* *}$ \\
ER fear & -.128 & $.186^{*}$ & $-.154^{*}$ \\
ER anger & .009 & $.170^{*}$ & -.078 \\
ER sadness & .079 & $.340^{* *}$ & -.268 \\
ER disgust & .018 & $.262^{* *}$ & $-.237^{* *}$ \\
PANSS P1 delusions in general & -.042 & .040 & .113 \\
PANSS P6 delusions of persecution & -.002 & .100 & .162 \\
PSYRATS delusional frequency & -.039 & $-.136^{*}$ & $.254^{* *}$ \\
PSYRATS delusional distress & -.053 & .003 & $.139^{*}$ \\
\hline
\end{tabular}

Notes: ER Emotion recognition, PANSS Positive and Negative Syndrom Scale, PSYRATS Psychotic Symptom Rating scale; PSQ therapy alliance Patient Session Questionnaire, TSQ therapy alliance Therapist session questionnaire; * $p<.05 ;{ }^{* *}: p<.01$

Table 4 Hierarchical multiple regression analyses predicting the quality of the therapeutic alliance and interactional problems

\begin{tabular}{|c|c|c|c|c|c|c|c|}
\hline Predictors & B & t & $\mathrm{p}$ & $F / F$ Change & df & $p$ & Adjusted $R^{2} / R^{2}$ change \\
\hline \multicolumn{8}{|l|}{ Regression 1: TSQ therapist alliance ${ }^{1}$} \\
\hline Step $1 * *$ & & & & 7.741 & 3168 & $<.0001$ & .106 \\
\hline PANSS total (covariate) & .011 & .144 & .886 & & & & \\
\hline Emotion Recognition** & .339 & 4.643 & $<.0001$ & & & & \\
\hline PSYRATS delusional frequency & -.110 & -1.399 & .164 & & & & \\
\hline Step $2 * *$ & & & & 7.406 & 1167 & .007 & .037 \\
\hline PANSS total (covariate) & -.018 & -.230 & .818 & & & & \\
\hline Emotion Recognition ${ }^{* *}$ & .288 & 3.881 & $<.0001$ & & & & \\
\hline PSYRATS delusional frequency & -.057 & -.715 & .475 & & & & \\
\hline Interaction ${ }^{* *}$ : & .205 & 2.721 & .007 & & & & \\
\hline \multicolumn{8}{|c|}{ Regression 2: TSQ interactional problems ${ }^{1}$} \\
\hline Step $1 * *$ & & & & 7.741 & 3168 & $<.0001$ & .106 \\
\hline PANSS total (covariate) $)^{* *}$ & .221 & 2.911 & .004 & & & & \\
\hline Emotion Recognition ** & -.252 & -3.565 & $<.0001$ & & & & \\
\hline PSYRATS delusional frequency* & .176 & 2.316 & .022 & & & & \\
\hline Step $2 * *$ & & & & 7.406 & 1167 & .007 & .139 \\
\hline PANSS total (covariate) ${ }^{* *}$ & .244 & 3.207 & .002 & & & & \\
\hline Emotion Recognition** & -.213 & -2.942 & .004 & & & & \\
\hline PSYRATS delusional frequency & -.135 & 1.749 & .0982 & & & & \\
\hline Interaction* & -.156 & -2.125 & .035 & & & & \\
\hline
\end{tabular}


negative impact of delusional frequency on TSQ therapy alliance.

Results of the second hierarchical regression analysis revealed a statistically significant model including ER and PSYRATS delusional frequency as predictors of TSQ interactional problems, but solely ER independently predicted interactional problems, whereas PSYRATS delusional frequency was not independently predictive of interactional problems. Including the interaction term in the second block resulted in a statistically significant model that explained a significant additional amount of variance in TSQ interactional problems and suggested a moderation effect.

The Johnson-Neyman technique revealed that ER moderated the negative influence of delusional frequency on TSQ interactional problems only in patients with ER scores (percentiles) between zero and 54.07\% (all $p<0.05$ ) suggesting that ER problems attenuated the negative impact of delusional frequency on TSQ interactional problems only in these patients. In persons with higher ratings, the moderation effect was not present.

\section{Discussion}

In comparison to controls, patients with psychosis presented problems in both ToM and emotion recognition (ER). Nevertheless, solely problems in ER were related to delusional distress, but not related to other measures of delusions. Problems in ER also had an impact on the quality of the therapeutic alliance and interactional problems viewed by the therapist. Finally, good ER ability reduced the negative impact of delusional frequency on both the quality of the therapeutic relationship and interactional problems during CBT/ST.

The present study is the first study reporting that problems in ER are associated with delusional distress, whereas there were no further associations with other measures of delusions. In particular, problems in recognizing the negative emotions fear and disgust were related with more pronounced delusional distress, whereas problems in recognizing the emotions anger and sadness were not related to delusional distress. Our results suggest that problems in ER (especially problems in recognizing fear and disgust) might not be directly related to the presence of delusions, but enhance the distress associated with them, in line with experimental designs suggesting that problems in ER are more pronounced in stressful situations in patients with psychosis [49]. It is possible that in stressful situations, patients with psychosis are less able to interpret social cues correctly and perform more errors in recognizing emotions and in inferring emotional states of other persons $[50,51]$ and this might lead to interactional problems and reduced social functioning [23]. Thus, it might be useful to further investigate using longitudinal assessments whether problems in ER - although not directly associated with the intensity of delusions - might increase delusional distress. In addressing this question, it is highly important to measure ER problems with regard to specific negative emotions, as our results demonstrate that problems in recognizing the emotions fear and disgust are related to delusional distress, whereas problems in recognizing anger and sadness were not related to more pronounced delusional distress. If longitudinal associations between problems in ER and specific negative emotions and delusional distress are further established, ER problems should then be included as one of the cognitive factors involved in the development and maintenance of delusional distress in theoretical models.

The fact that we did not find an association between ToM problems and any measure of delusions nor delusional frequency / distress is in line with findings of two meta-analyses $[14,18]$ and several other studies that did not report an association between ToM and delusions $[8,52,53]$, whereas negative symptoms and symptoms of disorganisation are more constantly associated with ToM problems [14]. One explanation might be that ToM problems are less pronounced in patients with delusions in comparison to patients with negative or disorganized symptoms (e.g. [54, 55], see Spronghorst et al. for a review on the literature of subgroup comparisons [56]) and thus, harder to assess using typical ToM paradigms that sometimes lack ecological validity [57]. Interestingly, all studies that used more ecologically valid ToM assessments such as movies of social situations (e.g. the Movie of Assessment of Social Cognition (MASC) [58] or the Movie Task of social situations [20]), found associations between ToM problems and more pronounced general and persecutory delusions in patients with psychosis [59].

An additional limitation of current ToM paradigms is the fact that they often measure ToM in a wright-orwrong format and thus investigate solely reduced ToM abilities/undermentalizing in patients with psychosis, whereas Abu-Akel $[60,61]$ suggested that patients with delusion rather present problems in overmentalizing / "hypermentalizing" mental states of other persons, defined as Hyper-ToM [58, 62]. First studies addressed the question of associations between Hyper-ToM and delusions in children with psychotic experiences and normal controls [63, 64] and patients with psychosis [58] and found evidence of an association. Thus, Hyper-ToM rather than undermentalizing might play an important role in the formation and maintenance of delusions and should be investigated in future studies.

Concluding, future studies that address the question of associations between ToM problems and delusions might be well-advised to use tasks with more pronounced ecological validity, for example, ToM assessment using 
videos, virtual reality (Virtual Assessment of Mentalising Ability (VAMA) [65] or investigating ToM problems in real-life using the experience sampling method [66]. Nevertheless, if there are still no associations between problems in Hyper-ToM and delusions, theoretical models correctly excluded ToM as one of the important cognitive factors involved in the formation and maintenance of delusions or positive symptoms [12, 13].

Our study is the first to report that therapists who treated patients with problems in ER (especially problems in recognizing the emotions disgust and fear) perceived more pronounced interactional problems in these patients. In addition, therapists who treated patients with problems in ER (especially in recognizing the emotions fear, anger, sadness and disgust) rated the quality of the therapeutic relationship more negatively. Our findings are partly in line with the study of Jung and colleagues [29] who reported an association between patients' ratings on the quality of the therapeutic alliance and ToM problems, but no associations between therapists' ratings and ToM problems, but the size of our study sample enabled us to detect associations of medium and small effect size. Nevertheless, it has to be taken into account, that our results were not pre-registered, but obtained in an exploratory analysis of a randomized-controlled therapy trial, thus, careful replication of our results should be performed, especially in light of the current replication crisis in psychology (see [67] for a review)). If our results are successfully replicated and patients' problems in ER influence the relationship with an empathetic and highly skilled therapist, it can be assumed that their problems in ER also have a negative impact on other social interactions in their daily life, as suggested by several other studies that directly addressed this question [21, 22].

In addition, we could provide evidence for the clinically important negative impact of delusional frequency on both the therapeutic relationship and interactional problems viewed by the therapist. Again, it is important to note that these results were obtained in an exploratory analysis and thus are in need of careful replication. If our findings are replicated in longitudinal preregistered assessments, they suggest that delusional frequency negatively affects social interactions (the therapeutic interactions) and thus may also partly contribute to the association between delusions and lower social functioning [68, 69], negative family atmosphere [70], more pronounced loneliness $[71,72]$ and social exclusion $[73,74]$.

Further, preserved ER abilities might protect patients from the negative influence of their delusions on the quality of the therapeutic relationship and interactional problems, as a statistically significant moderation effect occurred. Interestingly, the interaction was most pronounced in patients with severe ER problems: in this subgroup, problems in ER had a specific negative influence on both the therapeutic relationship and interactional problems. Our results are partly supported by a second study that addressed the impact of ToM on the association between delusions and self-rated social functioning [31]. Their results also suggest that preserved ToM abilities moderated the relationship between persecutory delusions and self-rated social functioning [31]. The fact that we did not find a similar moderation effect between ToM and the quality of the therapeutic relationship could be explained by different ToM assessments: the study used the Hinting task [54]) that is based on verbal descriptions of social situations, we used a picture sequencing test based on comics. To some degree, our findings expand on their results, as we used therapists' ratings of the therapeutic alliance as a direct measure of social functioning instead of self-ratings. Again, if our exploratory findings can be replicated and positive ER skills are a protecting factor against the negative influence of delusions on the therapeutic relationship, patients' ER abilities might also influence the effectiveness of CBT for psychosis, as a positive therapeutic relationship is closely related to the effectiveness of CBT [28, 75, 76]. Interestingly, one study indeed found general ToM abilities (including ER) to moderate change in positive symptoms in CBT [77]. Thus, interventions that improve ER and ToM abilities might be beneficial in order to improve the therapeutic relationship and, further, the effectiveness of CBT.

ER and ToM problems in patients with psychosis can also be viewed in a broader perspective as parts of patients' more general problems in their metacognitive capacities. Metacognition has been defined as "cognition about cognition" by Flavell [78] and also discussed as an important part of social cognition [79]. Both ToM and ER are important parts of metacognition, in combination with self-reflectivity (comprehension of one's own mental state), decentration (the ability to from a complex representation of the world) and mastery, the ability to use information of one's own and other mental states in respond to and to solve social and psychological problems [80].

In comparison to controls, patients with psychosis were found to present problems in almost all parts of metacognition (see Lysaker et al. [80] for a review on metacognition in schizophrenia). Metacognitive abilities in patients with psychosis are closely linked to a positive therapeutic relationship viewed by patients in CBT (mastery [81]) and also with positive outcome in Cognitive Remediation therapy (learning potential: [82]). Thus, as ER is an important part of metacognition, it is plausible that we also found a link between problems in ER and a 
less favourable therapeutic relationship and interactional problems viewed by the therapist, as ER can be viewed as one part of metacognitive mastery that was also found to be linked to a positive therapeutic relationship [81]. Thus, the association between problems in ER and a less favourable therapeutic relationship could be moderated by general metacognitive deficits in patients with psychosis. Therapists might perceive these deficits during the first therapeutic sessions and these problems might influence the therapeutic relationship.

For example, patients with deficits in ER and metacognition might present problems in their metacognitive self-reflection that could become visible in diagnostic sessions, as they might not be able to talk about their individual thoughts and emotions in specific situations. They also might present problems in understanding the basic cognitive model that consists of relations between individual perceptions, thoughts, emotions and behaviour [83] due to their problems in self-reflection. Further, patients might also present problems in decentration and thus might not be able to form a complex representation of the world that is important in therapy in order to solve personal and interpersonal problems, e.g. due to their well-known jumping-to-conclusions-bias [84]. Finally, patients' level of mastery in using their information on mental states in order to solve real-world problems might also be reduced.

Concluding, it is possible that the association between poor emotion recognition and the therapeutic relationship/interactional problems viewed by the therapist can be explained by patients' metacognitive problems. In addition, it is plausible that not only ER abilities but also a positive metacognitive performance might moderate the influence of delusional frequency on the therapeutic relationship and thus, might also be helpful for patients with psychosis in their general social life, as suggested by a study that found metacognitive capacities to mediate the negative influence of neurocognitive deficits on social functioning in patients with psychosis [85]. Thus, future studies will be well-advised to address all aspects of metacognition in patients with psychosis and their influence on the therapeutic relationship.

Our results suggest for therapists of patients with psychosis to take patients' potential ER problems (and their metacognitive deficits) into account in CBT for psychosis. First, it could be useful to assess patients with regard to their ER abilities before start of therapy. Second, if patients present ER problems, it is important for therapists to make a special effort to improve the therapeutic relationship with these patients. Third, it could be helpful to train ER (and metacognition) in patients with psychosis using specialized interventions from several social cognition trainings in the framework of Cognitive Remediation [86]: the Social Cognition and Interaction Training [87] and the Metacognitive Training (Moritz and Woodward [88]) aim on improving both ToM and ER, whereas the Training of Affect Recognition [41] aims more closely on ER. In general, these trainings were able to enhance both ToM and ER abilities [87, 89-91] and their general positive effect on social functioning is large [92, 93]. It is also possible, that an integration in or a combination of these trainings with $\mathrm{CBT}$ in order to improve ER might be beneficial.

\section{Strength and limitations}

Strengths of the present study include the large sample of patients with psychosis and the detailed assessment of different dimensions of delusions. An additional strength is the longitudinal assessment of the quality of the therapeutic relationship over five sessions.

In interpreting our findings, it should be mentioned that all associations between ToM, ER, delusions and the therapeutic relationship were of small effect size according to Cohen [94]. In addition, it should be noted that solely two of the four scientific hypotheses were pre-specified, whereas all associations between ER, ToM, delusions and the therapeutic relationship were of exploratory nature. Thus, the question of associations between ToM, ER and the therapeutic relationship and the moderation effect require an additional careful replication study.

In addition, while our moderation model and the mode of assessment (ER and delusions were assessed before the start of therapy) suggest a causal association between problems in ER and delusional frequency and also implicate that positive ER abilities moderate the association between delusional frequency and the therapeutic relationship, we cannot rule out the possibility that a low quality of the therapeutic relationship and interactional problems were influenced by other factors, e.g. common therapeutic factors such as therapists' empathy, expertness, attractiveness and trustworthiness [95, 96] and thus, it is also possible that an unfavourable therapeutic relationship might lead to more pronounced delusions in patients with psychosis. Thus, future studies should focus on symptom change, ER and the therapeutic relationship using multiple assessments in order to address the question whether more pronounced ER problems cause more pronounced delusional frequency/ distress and a less favourable therapeutic relationship (or vice versa).

Finally, it should be mentioned that the patients in the present study were patients interested in participating in a therapy trial who might present better general cognitive and social functioning and less pronounced problems in their social cognition. Nevertheless, as the moderation effect occurred predominantly 
in patients with lower ER skills, a potential selection bias might not influence the generalisation of our exploratory results.

\section{Conclusions}

Several important conclusions can be drawn from our results. First, problems in ER were related to enhanced delusional distress in patients with psychosis. In addition, patients' deficits in ER had a negative impact on the quality of the relationship viewed by their therapists. Nevertheless, positive ER in patients with psychosis moderated (reduced) the negative impact of delusions on the therapeutic relationship. Thus, improving ER might be a way to improve the quality of the therapeutic relationship and potentially the effectiveness of CBT or other interventions for patients with psychosis.

\section{Abbreviavions}

CBT: Cognitive Behavior Therapy:; CBTp: Cognitive Behavior Therapy for positive symptoms of psychosis.; DSM-IV: Diagnostic and Statistical Manual of Mental Disorders, 4th Edition.; ER: Emotion Regulation.; fMRI: functional Magnetic Resonance Imaging.; MWT-B: Mehrfachwahlwortschatztest (German verbal intelligence test).; PANSS: Positive and Negative Syndrome Scale.; PFA: Pictures of Facial Affect Test.; PSQ: Patient Session Questionnaire.; PSYRATS: Psychotic Symptoms Rating Scale.; ST: Suppportive Therapy:; ToM: Theory of Mind.; TSQ: Therapist Session Questionnaire.

\section{Acknowledgements}

Gudrun Sartory, one of the initiators and principal investigators of the present study, sadly passed away in 2015. We owe much to her leadership, optimism, and lasting support. We would like to thank Jürgen Brinkmeyer for programming the ER task. We would like to thank all participants in the study and all members of the POSITIVE study: S. Baal, J. Berning, S. Beulen, G. Buchkremer, A. Büch, B. Conradt, Y. Eikenbusch, W. Gaebel, A. Gawronski, J. Güttgermans, A. Herold, U. Jakobi-Malterre, I. Lengsfeld, W. Maier, K. Platt, B. Pohlmann, A. Rotarska-Jagiela, S. Sickinger, H. Smoltczyk, S. Unsöld, A. Vogeley, A. Witt, L. Zipp.

\section{Authors' contribution}

$A B, J H, T K, S K, B M, G W, A W, W W$ and MW designed the study and wrote the protocol and the trial proposal, SM, A-CS and ML performed the statistical analysis, SM and A-C S managed the literature search and wrote the first draft of the manuscript, MW, $\mathrm{KH}$ and DS co-wrote the manuscript. All authors contributed to and have approved the final manuscript.

\section{Funding}

This work was supported by grants awarded by the German Federal Ministry of Education and Research (BMBF: 01GV0618, 01GV0620). The study was part of the BMBF research program "Research Networks on Psychotherapy." The German Federal Ministry of Education and Research was neither involved in the design of the study, the data collection, the data analysis, in the interpretation of data nor in writing the manuscript.

\section{Availability of data and materials}

The dataset is available from the corresponding author on reasonable request.

\section{Ethics approval and consent to participate}

All participants were informed about the assessment and gave written informed consent. In case of a legal guardian, the patients and the guardian were informed about the assessment and both the patient and the guardian gave written informed consent. The ethics committees at the six centres' medical faculties (University of Bonn, Cologne, Duesseldorf, Frankfurt, Tuebingen and Duisburg-Essen) approved the study.
Consent for publication

Not applicable.

\section{Competing interests}

SM, AB, KH, SK, TK, AW and WW have published manuals/ chapters of textbooks and presented workshops and supervision in CBT (all) and cognitive remediation (WW) for psychosis. All authors declare that they have no competing interests.

\section{Author details}

${ }^{1}$ Department of Psychiatry and Psychotherapy, University of Bonn, Sigmund-Freud-Straße 25, 53105 Bonn, Germany. ${ }^{2}$ Department of Psychiatry and Psychotherapy, Philipps-University of Marburg, Rudolf-Bultmann-Straße 8 , 35039 Marburg, Germany. ${ }^{3}$ Department of Psychiatry and Psychotherapy, University of Tübingen, Calwerstraße 14, 72076 Tübingen, Germany. ${ }^{4}$ Department of Psychiatry and Psychotherapy, University of Cologne, Gleuler Straße, 50931 Köln, Germany. ${ }^{5}$ Department of Psychiatry and Psychotherapy, Vivantes Hospital Berlin, Dieffenbachstraße 1, 10967 Berlin, Germany.

${ }^{6}$ Department of Psychiatry, Psychosomatic and Psychotherapy, University of Frankfurt, Heinrich-Hoffmann-Straße 10, 60528 Frankfurt am Main, Germany. ${ }^{7}$ Department of Psychiatry and Psychotherapy, University of Duisburg- Essen, Virchowstraße 147, 45147 Essen, Germany. ${ }^{8}$ Department of Psychiatry and Psychotherapy, Hospital Fulda, Pacelliallee 4, 36043 Fulda, Germany.

${ }^{9}$ Department of Psychiatry and Psychotherapy, Medical Faculty,

Heinrich-Heine University of Düsseldorf, Bergische Landstraße 2, 40629

Düsseldorf, Germany.

Received: 12 October 2019 Accepted: 4 February 2020

Published online: 10 February 2020

\section{References}

1. Premack D, Woodruff G. Does the chimpanzee have a 'theory of mind'? Behav Brain Sci. 1978;4:515-26.

2. Adolphs R. The neurobiology of social cognition. Curr Opin Neurobiol. 2001; 11(2):231-9.

3. Green MF, Leitmann DI. Social cognition in schizophrenia. Schizophr Bull. 2008:34:670-2.

4. Frith CD. The cognitive neuropsychology of schizophrenia. Hove: Lawrence Erlbaum Associates; 1992.

5. Bora $E$, Yucel M, Pantelis C. Theory of mind impairment in schizophrenia: meta-analysis. Schizophr Res. 2009;109(1-3):1-9.

6. Bora $\mathrm{E}$, Pantelis $\mathrm{C}$. Theory of mind impairments in first-episode psychosis, individuals at ultra-high risk for psychosis and in first-degree relatives of schizophrenia: systematic review and meta-analysis. Schizophr Res. 2013; 144(1):31-6

7. Harrington L, Langdon R, Siegert R, Mc CJ. Schizophrenia, theory of mind, and persecutory delusions. Cogn Neuropsychiatry. 2005;10:87-104.

8. Greig T, Bryson GJ, Bell MD. Theory of mind performance in schizophrenia: diagnostic, symptom and neuropsychological correlates. J Nerv Ment Dis. 2004;192:12-8.

9. Bentall RP, de Sousa P, Varese F, Wickham S, Sitko K, Haarmans M, et al. From adversity to psychosis: pathways and mechanisms from specific adversities to specific symptoms. Soc Psychiatry Psychiatr Epidemiol. 2014; 49(7):1011-22

10. Preti A, Cella M. Paranoid thinking as a heuristic. Early Interv Psychiatry. 2010;4(3):263-6.

11. Garety P, Bebbington P, Fowler D, Freeman D, Kuipers E. Implications for neurobiological research of cognitive models of psychosis: a theoretical paper. Psychol Med. 2007;37(10):1377-91.

12. Freeman D, Garety P. Advances in understanding and treating persecutory delusions: a review. Soc Psychiatry Psychiatr Epidemiol. 2014:49(8):1179-89.

13. Garety $P$, Freeman D. The past and future of delusion research: from the inexplicable to the treatable. Br J Psychiatry. 2013;203:327-33.

14. Ventura J, Wood RC, Hellemann GS. Symptom domains and neurocognitive functioning can help differentiate social cognitive processes in schizophrenia: a meta-analysis. Schizophr Bull. 2013;39(1):102-11.

15. Mitchell RLC, Phillips LH. The overlapping relationship between emotion perception and theory of mind. Neuropsychologia. 2015;70:1-10.

16. Kohler CG, Walker JB, Martin EA, Healey KM, Moberg PJ. Facial emotion perception in schizophrenia: a meta-analytic review. Schizphrenia Bulletin. 2010;36(5):1009-19. 
17. Chan RC, Li H, Cheung EF, Gong QY. Impaired facial emotion perception in schizophrenia: a meta-analysis. Psychiatry Res. 2010;178(2):381-90.

18. Ventura J, Wood RC, Jimenez AM, Hellemann GS. Neurocognition and symptoms identify links between emotion recognition and emotion processing in schizophrenia: meta-analytic findings. Schizophr Res. 2013; 151(0):78-84.

19. Gaweda L, Staszkiewicz M, Balzan R. The relationship between cognitive biases and psychological dimensions of delusions: the importance of jumping to conclusions. J Behav Ther Exp Psychiatry. 2017;56:51-6.

20. Mehl S, Rief W, Mink K, Lüllmann E, Lincoln TM. Social performance is more closely associated with theory of mind and autobiographical memory than with psychopathological symptoms in clinically stable patients with schizophrenia-spectrum disorders. Psychiatry Res. 2010;178(2):276-83.

21. Gur RE, Kohler CG, Ragland JD. Flat affect in schizophrenia: relation to emotion processing and neurocognitive measures. Schizophr Bull. 2006;32: 279-87

22. Riehle M, Mehl S, Lincoln TM. The specific social costs of expressive negative symptoms in schizophrenia; reduced smiling predicts interactional outcome. Acta Psychiatr Scand. 2018;138(2):133-44.

23. Fett AK, Viechtbauer W, Dominguez MD, Penn DL, van Os J, Krabbendam L. The relationship between neurocognition and social cognition with functional outcomes in schizophrenia: a meta-analysis. Neurosci Biobehav Rev. 2011;35(3):573-88.

24. Ventura J, Hellemann GS, Thames AD, Koellner V, Nuechterlein KH. Symptoms as mediators of the relationship between neurocognition and functional outcome in schizophrenia: a meta-analysis. Schizophr Res. 2009; 113(2-3):189-99.

25. Martin DJ, Garske JP, Davis MK. Relation of the therapeutic alliance with outcome and other variables: a meta-analytic review. J Consult Clin Psychol. 2000;68(3):438-50.

26. Horvath $A O$, Del Re AC, Flückinger C, Symonds D. Alliance in individual psychotherapy. In: Norcross JC, editor. Psychotherapy relationships that work evidence-based responsiveness. 2nd ed. Oxford: University Press; 2011. p. 25-69.

27. Flückinger $C$, Del Re AC, Wampold BE, Symonds D, Horvath AO. How central is the alliance in psychotherapy? A multilevel longitudinal meta-analysis. J Couns Psychol. 2012;59:1-17.

28. Shattock L, Berry K, Degnan A, Edge D. Therapeutic alliance in psychological therapy for schizophrenia and related psychosis: a systematic review. Clin Psychol Psychother. 2017;25:60-85.

29. Jung E, Wiesjahn M, Lincoln TM. Negative, not positive symptoms predict the early therapeutic alliance in cognitive behavioral therapy for psychosis. Psychother Res. 2013;24(2):171-83.

30. Wittorf A, Jakobi-Malterre UE, Bechdolf A, Mueller BW, Sartory G, Wagner M, et al. The influence of baseline symptoms and insight on the therapeutic alliance early in the treatment of schizophrenia. Eur Psychiatry. 2009;24:259-67.

31. Phalen PL, Dimaggio G, Popolo R, Lysaker P. Aspects of theory of mind that attenuate the relationship between persecutory delusions and social functioning in schizophrenia spectrum disorders. J Behav Ther Exp Psychiatry. 2017;65:65-70.

32. Klingberg S, Wittorf A, Meisner C, Wölwer W, Wiedemann G, Herrlich J, et al. Cognitive behavioural therapy versus supportive therapy for persistent positive symptoms in psychotic disorders: the POSITIVE study, a multicenter, prospective, single-blind, randomised controlled clinical trial. Trials. 2010;11:123

33. Wittchen HU, Wunderlich U, Gruschwitz S, Zaudig M. Strukturiertes Klinisches Interview fuer DSM-VI (SKID). Göttingen: Hogrefe; 1997.

34. Kay SR, Opler LA, Fiszbein A. Positive and negative syndrome scale (PANSS). New York: Multi Health Systems Inc:; 1992.

35. Lehrl S. Mehrfachwahl-Wortschatz-Intelligenztest MWT-B. Fünfte unveränderte Auflage. Spitta Verlag: Balingen; 2005.

36. Walter H, Adenzato M, Ciaramidaro A, Enrici I, Pia L, Bara BG. Understanding intentions in social interaction: the role of the anterior paracingulate cortex. J Cogn Neurosci. 2004;16(10):1854-63.

37. Walter H, Ciaramidaro A, Adenzato M, Vasic N, Ardito RB, Erk S, et al. Dysfunction of the social brain in schizophrenia is modulated by intention type: an fMRI study. Soc Cogn Affect Neurosci. 2009;4(2):166-76.

38. Monke S, Erk S, Schnell K, Schütz C, Romanczuk-Seiferth N, Grimm O, et al. Further evidence for the impact of a genome-wide-supported psychosis risk variant in ZNF804A on the theory of mind network. Neuropsychopharmacology. 2014;39(5):1196-205.
39. Ekman P, Friesen WV. Pictures of facial affect. Palo Alto: Consulting Psychologist Press; 1976

40. Woelwer W, Frommann N, Halfmann S, Piaszek A, Streit M, Gaebel W. Remediation of impairments in facial affect recognition in schizophrenia: efficacy and specificity of a new training program. Schizophr Res. 2005;80: 295-303.

41. Frommann N, Streit M, Woelwer W. Remediation of facial affect recognition impairments in patients with schizophrenia: a new training program. Psychiatry Res. 2003;117:281-4.

42. Haddock G, McCarron J, Tarrier N, Faragher EB. Scales to measure dimensions of hallucinations and delusions: the psychotic symptom rating scales (PSYRATS). Psychol Med. 1999;29(4):879-89.

43. Woodward TS, Jung K, Hwang H, Yin J, Taylor L, Menon M, et al. Symptom dimensions of the psychotic rating scales in psychosis: a multisite study. Schizophr Bull. 2014:40(4):265-74

44. Flückiger C, Regli D, Zwahlen D, Hostettler S, Caspar F. Der Berner Patienten- und Therapeutenstundenbogen 2000. Z Klin Psychol Psychother. 2010;39(2):71-9

45. Uttl B. Measurement of individual differences: lessons from memory assessment in research and clinical practice. Psychol Sci. 2005;16(6):460-7.

46. Berres M, Zehnder A, Bläsi S, Monsch AU. Evaluation of diagnostic scores with adjustment for covariates. Stat Med. 2008;27(10):1777-90.

47. Hayes AF, Preacher KJ. Statistical mediation analysis with a multicategorical independent variable. Br J Math Stat Psychol. 2014;67:451-70.

48. Bauer DJ, Curran PJ. Probing interactions in fixed and multilevel regression: inferential and graphical techniques. Multivar Behav Res. 2005;40(3):373-400.

49. Köther U, Lincoln TM. Moritz S. Psychiatry Research: Emotion perception and overconfidence in errors under stress in psychosis; 2018.

50. Lincoln TM, Reumann R, Moritz S. Is there a functional way of responding to paranoid intrusions? Development of the reactions to paranoid thoughts scale. Cogn Neuropsychiatr. 2010;15(4):377-96.

51. Freeman D, Stahl D, McManus S, Meltzer H, Brugha T, Wiles $N$, et al. Insomnia, worry, anxiety and depression as predictors of the occurrence and persistence of paranoid thinking. Soc Psychiatry Psychiatr Epidemiol. 2012;47(8):1195-203.

52. Langdon R, Coltheart M, Ward P. Empathetic perspective-taking is impaired in schizophrenia: evidence from a study of emotion attribution and theory of mind. Cogn Neuropsychiatr. 2006;11(2):133-55.

53. Langdon R, Coltheart M, Ward PB, Catts SV. Mentalising, executive planning and disengagement in schizophrenia. Cogn Neuropsychiatr. 2001;6(2):81-108.

54. Corcoran R, Mercer M, Frith C. Schizophrenia, symptomatology and social inference: investigating theory of mind in people with schizophrenia. Schizophr Res. 1995;17:5-13.

55. Frith CD, Corcoran R. Exploring 'theory of mind' in people with schizophrenia. Psychol Med. 1996;26:521-30.

56. Sprong M, Schothorst $P$, Vos E, Hox J, van Engeland $H$. Theory of mind in schizophrenia. Br J Psychiatry. 2007;191:8-13.

57. Byom LJ, Mutlu B. Theory of mind: mechanisms, methods and new directions. Front Hum Neurosci. 2013;7:413.

58. Montag C, Dziobek I, Richter IS, Neuhaus K, Lehmann A, Sylla R, et al. Different aspects of theory of mind in paranoid schizophrenia: evidence from a video-based assessment. Psychiatry Res. 2011:186(2-3):203-9.

59. Mehl S, Rief W, Lüllmann E, Ziegler M, Kesting ML, Lincoln TM. Are theory of mind deficits in understanding intentions of others associated with persecutory delusions? J Nerv Ment Dis. 2010;198(7):516-9.

60. Abu-Akel A, Bailey A. The possibility of different forms of theory of mind impairment in psychiatric and developmental disorders. Psychol Med. 2000; 30(3):735-8.

61. Abu-Akel A. Impaired theory of mind in schizophrenia. Pragmat Cogn. 1999;7(2): 247-82.

62. Fretland A, Andersson S, Sundet K, Andreassen OA, Melle I, Vaskinn A. Theory of mind in schizophrenia: error types and associations with symptoms. Schizophr Res. 2015;162(1-3):42-6.

63. Clemmensen L, van Os J, Skovgaard AM, Væver M, Blijd-Hoogewys EM, Bartels-Velthuis AA, Jeppesen P. Hyper-theory-of-mind in children with psychotic experiences. Plos one. 2014;9(11).

64. Clemmensen L, van Os J, Drukker M, Munkholm A, Rimvall MK, Vaever M, et al. Psychotic experiences and hyper theory-of-mind in preadolescence - a birth cohort study. Psychol Med. 2016;46(1):87-101.

65. Canty AL, Neumann DL, Fleming J, Shum DHK. Evaluation of a newly developed measure of theory of mind: the virtual assessment of mentalising ability. Neuropsychol Rehabil. 2015;27(5):834-70. 
66. Bryant L, Coffey A, Povinelli DJ, Pruett JR. Theory of mind experience sampling in typical adults. Conscious Cogn. 2013;22:697-707.

67. Shrout PE, Rodgers JL. Psychology, science, and knowledge construction: broadening perspectives from the replication crisis. Annu Rev Psychol. 2018; 69:487-510.

68. Schaub D, Brüne M, Jaspen E. G. PF, Bierhoff HW, Juckel G. the illness and everyday living: close interplay of psychopathological syndromes and psychosocial funtioning in chronic schizophrenia. Eur Arch Psychiatry Clin Neurosci. 2011;261(2):85-93.

69. Dickson JM, Barsky J, Kinderman P, King D, Taylor PJ. Early relationship and paranoia: qualitative investigation of childhood experiences associated with the development of persecutory delusions. Psychiatry Res. 2016;238:40-5.

70. Hesse K, Kriston L, Mehl S, Wittorf A, Wiedemann W, Wölwer W, et al. The vicious cycle of family atmosphere, interpersonal self-concepts, and paranoia in schizophrenia—a longitudinal study. Schizophr Bull. 2015;41(6): 1403-12.

71. Meltzer H, Bebbington P, Dennis MS, Jenkins R, McManus S, Brugha TS Feelings of loneliness among adults with mental disorder. Soc Psychiatry Psychiatr Epidemiol. 2013;48(1):5-13.

72. Kimhy D, Delespaul P, Corcoran C, Ahn H, Yale S, Malaspina D. Computerized experience sampling method (ESMc): assessing feasibility and validity among individuals with schizophrenia. J Psychiatr Res. 2006;40(3): 221-30.

73. Sündermann O, Onwumere J, Kane F, Morgan C, Kuipers E. Social networks and support in first-episode psychosis: exploring the role of loneliness and anxiety. Soc Psychiatry Psychiatr Epidemiol. 2014;49(3):359-66.

74. Giacco D, Palumbo C, Strappelli N, Catapano F, Priebe S. Social contacts and loneliness in people with psychosis. Compr Psychiatry. 2016;70:190-9.

75. Frank AF, Gunderson JG. The role of the therapeutic alliance in the treatment of schizophrenia. Relationship to course and outcome. Arch Gen Psychiatry. 1990;47:228-36.

76. Priebe S, Richardson M, Cooney M, Adedeji O, Mc CR. Does the therapeutic relationship predict outcomes of psychiatric treatment in patients with psychosis? A systematic review. Psychother Psychosom. 2011;80:70-7.

77. Lincoln TM, Rief W, Westermann S, Ziegler M, Kesting ML, Heibach E, et al. Who stays, who benefits? Predicting dropout and change in cognitive behaviour therapy for psychosis. Psychiatry Res. 2014;216(2):198-205.

78. Flavell JH. Metacognition and cognitive monitoring: a new area of cognitive-development inquiry. Am Psychol. 1979;34(10):906-11.

79. Pinkham AE, Penn DL, Green MF, Buck B, Healey K, Harvey PD. The social cognition psychometric evaluation study: results of the expert survey and RAND panel. Schizophr Bull. 2014;40:813-23.

80. Lysaker P, Vohs J, Minor KS, Irrazaval L, Leonhardt B, Hamm JA, et al. Metacognitive deficits in schizophrenia: presence and association with psychosocial outcome. J Nerv Ment Dis. 2015;203:530-6.

81. Davies LW, Eicher AC, Lysaker P. Metacognition as a predictor of the therapeutic alliance over 26 weeks of psychotherapy in schizophrenia. Schizophr Res. 2011;129:85-90.

82. Tas C, Brown EC, Esen-Danaci A, Lysaker P, Bruene M. Intrinsic motivation and metacognition as predictors of learning potential in patients with remitted schizophrenia. J Psychiatr Res. 2012;46:1086-92.

83. Beck JS. Cognitive behavior therapy: basics and beyond. New York: Guilford; 2011.

84. Ross RM, Mc Kay R, Coltheart M, Langdon R. Jumping to conclusions about the beads task? A meta-analysis of delusional ideation and data-gathering Schizophr Bull. 2015;41(5):1183-91.

85. Lysaker P, Shea AM, Buck KD, Dimmagio G, Procacci M, Salvatore G, et al. Metacognition as a mediator of the effects of impairment in neurocognition on social functioning in schizophrenia spectrum disorders. Acta Psychologica Scandinavica. 2010;122:405-13.

86. Wykes T, Huddy V, Cellard C, McGurk SR, Czobor P. A meta-analysis of cognitive remediation for schizophrenia: methodology and effect sizes. Am J Psychiatr. 2011;168(5):472-85.

87. Combs DR, Adams SD, Penn DL, Roberts D, Tiegreen J, Stem P. Social cognition and interaction training (SCIT) for inpatients with schizophrenia spectrum disorders: preliminary findings. Schizophr Res. 2007;91(1-3):112-6.

88. Moritz S, Woodward TS. Metacognitive training in schizophrenia: from basic research to knowledge translation and intervention. Curr Opin Psychiatry. 2007;20(6):619-25.

89. Kowalski J, Pankowski D, Lew-Starowicz M, Gawęda L. Do specific metacognitive training modules lead to specific cognitive changes among patients diagnosed with schizophrenia? A single module effectiveness pilot study. Psychosis. 2017:9(3):254-9.

90. Grant N, Lawrence M, Preti A, Wykes T, Cella M. Social cognition interventions for people with schizophrenia: a systematic review focussing on methodological quality and intervention modality. Clin Pschol Rev. 2017; 56:55-64.

91. Vaskinn A, Lovgren A, Egeland MK, Feyer FK, Ostefjells T, Andreassen OA, et al. A randomized controlled trial of training of affect recognition (TAR) in schizophrenia showing lasting effects for theory of mind. 2019.

92. Kurtz MM, Richardson CL. Social cognitive training for schizophrenia: a meta-analytic investigation of controlled research. Schizophr Bull. 2012;38(5): 1092-104.

93. Kurtz MM, Gagen E, Rocha NBF, Machado S, Penn DL. Comprehensive treatments for social cognitive deficits in schizophrenia: a critical review and effect-size analysis of controlled studies. Clin Psychol Rev. 2016:43:80-9.

94. Cohen D. Statistical power for the social sciences. Hillsdale: Erlbaum; 1992.

95. Evans-Jones C, Peters E, Barker C. The therapeutic relationship in CBT for psychosis: client, therapist and therapy factors. Behav Cogn Psychother. 2009;37(5):527-40

96. Jung E, Wiesjahn M, Rief W, Lincoln TM. Perceived therapist genuineness predicts therapeutic alliance in cognitive behavioural therapy for psychosis. Br J Clin Psychol. 2015:54:34-48.

\section{Publisher's Note}

Springer Nature remains neutral with regard to jurisdictional claims in published maps and institutional affiliations.
Ready to submit your research? Choose BMC and benefit from:

- fast, convenient online submission

- thorough peer review by experienced researchers in your field

- rapid publication on acceptance

- support for research data, including large and complex data types

- gold Open Access which fosters wider collaboration and increased citations

- maximum visibility for your research: over $100 \mathrm{M}$ website views per year

At $\mathrm{BMC}$, research is always in progress.

Learn more biomedcentral.com/submissions 